\title{
Which Predicts Quadriceps Muscle Strength in Knee Osteoarthritis: Biological Markers or Clinical Variables?
}

\author{
Neslihan GÖKÇEN, ${ }^{1}$ Sibel BAŞARAN, ${ }^{2}$ İlke Coşkun BENLİDAYI, ${ }^{2}$ \\ Çiğdem ÖZDEMİR, ${ }^{3}$ Gülş̧ah SEYDAOĞLU ${ }^{4}$ \\ ${ }^{1}$ Department of Physical Medicine and Rehabilitation, Division of Rheumatology, Medical Faculty of Çukurova University, Adana, Turkey \\ ${ }^{2}$ Department of Physical Medicine and Rehabilitation, Medical Faculty of Çukurova University, Adana, Turkey \\ ${ }^{3}$ Department of Physiology, Medical Faculty of Çukurova University, Adana, Turkey \\ ${ }^{4}$ Department of Biostatistics, Medical Faculty of Çukurova University, Adana, Turkey
}

\begin{abstract}
Objectives: This study aims to evaluate the relationship between biological markers and quadriceps muscle strength, the correlation of clinical variables with quadriceps muscle strength, and the results according to the radiological severity in patients with knee osteoarthritis.

Patients and methods: A total of 152 patients ( 22 males, 130 females; mean age $57.3 \pm 7.5$ years; range 40 to 70 years) with primary knee osteoarthritis were included in the study. We evaluated biological markers of C-telopeptide of type I collagen, C-telopeptide of type II collagen, leptin, and osteocalcin along with 25 -hydroxy vitamin $\mathrm{D}$. We measured quadriceps muscle strength both by manual muscle tester and computerized isokinetic dynamometer. We evaluated pain and functional status of the patients by visual analog scale and Western Ontario and McMaster Universities Osteoarthritis Index. We analyzed the correlation between biological markers and quadriceps muscle strength along with clinical variables. We classified the strength of correlations as no-very weak, weak-moderate, moderate-strong, and excellent.

Results: Of the patients, $76.9 \%(n=117)$ were obese. Quadriceps muscle strength measures were significantly lower in females than that in males. There was no-very weak correlation between biological marker levels and quadriceps muscle strength. However, weak-moderate correlations were found between clinical variables (pain and Western Ontario and McMaster Universities Osteoarthritis Index scores) and quadriceps muscle strength measures.

Conclusion: Among the measured biological markers, none had any influence on quadriceps muscle strength in patients with knee osteoarthritis. However, pain and functional status of the patients might affect quadriceps muscle strength.

Keywords: Biological markers; knee osteoarthritis; quadriceps muscle strength; vitamin D.
\end{abstract}

Osteoarthritis (OA), the most common joint disorder, affects not only the joint cartilage, but also other joint structures such as subchondral bone, joint capsule, and quadriceps muscle..$^{1-3}$ Since both cartilage and other joint structures are affected from $\mathrm{OA}$, research on quadriceps muscle strength, bone, and cartilage biomarkers has been carried out during recent years. ${ }^{4,5}$ These biomarkers are thought to be a predictor for early $\mathrm{OA}$ and to determine the prognosis of OA. Although the relationship of biological markers with radiological severity has been investigated in several studies, ${ }^{6-9}$ evidence regarding the relationship between biomarkers and quadriceps muscle strength in patients with knee $\mathrm{OA}$ is scarce.

In this study, we hypothesized that quadriceps muscle strength is diminished in knee OA and questioned whether this could be determined by biological markers, clinical variables, and radiological severity. In accordance with this hypothesis, in this study, we aimed to evaluate the relationship between biological markers and 
quadriceps muscle strength, the correlation of clinical variables with quadriceps muscle strength, and the results according to the radiological severity in patients with knee OA.

\section{PATIENTS AND METHODS}

The study consisted of 152 patients (22 males, 130 females; mean age $57.3 \pm 7.5$ years; range 40 to 70 years) with primary knee OA fulfilling the clinical and radiological diagnostic criteria of American College of Rheumatology $y^{1}$ and was performed in a cross-sectional design at the outpatient clinic of a university hospital between May 2013 and April 2014. Exclusion criteria were as follows: (i) history of any surgery to the lower extremity, (ii) history of malignancy, (iii) presence of neurological diseases including hemiplegia, paraplegia and/or polio sequela, (iv) presence of inflammatory diseases such as rheumatoid arthritis, ankylosing spondylitis and/or reactive arthritis, ( $v$ ) diagnosis of septic arthritis, (vi) diagnosis of crystal arthropathy, (vii) presence of any cardiac diseases.

We recorded the demographic features and clinical evaluation of the individuals according to a standard form. We evaluated knee pain by using visual analog scale. We used the Western Ontario and McMaster Universities (WOMAC) OA Index (3.1 questionnaire), valid in our population, to assess functional status. ${ }^{10}$ Besides, we filled out a comorbidity questionnaire for all patients. ${ }^{11}$

We invited the patients to the outpatient clinic to perform the isokinetic evaluation. We obtained blood samples from each patient on the same day of isokinetic testing. The examiner who conducted the isokinetic test was blinded to the results of the laboratory analysis regarding the biomarkers.

We investigated the biomarkers of the cartilage, adipose tissue, and bone. Additionally, as vitamin $\mathrm{D}$ deficiency is related with muscle weakness, we measured the 25-hydroxy vitamin D [25-(OH)D] level.

We measured the C-telopeptide of type II collagen (CTX-II) level in serum via human CTX-II enzyme-linked immunosorbent assay (ELISA) kit (Elabscience) by using sandwich-ELISA method at $450 \mathrm{~nm}$. We measured the leptin level in serum via human leptin ELISA kit (Biovendor) by using sandwich-ELISA method at $450 \mathrm{~nm}$ and $630 \mathrm{~nm}$. We measured both C-telopeptide of type I collagen (CTX-I) and osteocalcin levels via electrochemiluminescence immunoassay method. We measured the 25-(OH)D-level in serum was measured by using high performance liquid chromatography method.

We evaluated the strength of the quadriceps muscle using the two below-mentioned methods:

1) Manual muscle test: Manual muscle tester (MMT) is a portable system that evaluates isometric muscle strength and range of motion. In the present study, we used the microFET3 (Hoggan Health Industries) MMT device to measure quadriceps muscle strength and noted the results as pound $(1 \mathrm{lb}=0.45 \mathrm{~kg})$.

2) Isokinetic test: We measured the peak torque of knee extensor and flexor muscles by isokinetic dynamometer (NORM 6000 CSMI, USA) on isometric and isokinetic module. Prior to the isometric measurement, we seated the patients on the dynamometric chair and stabilized them with waist and chest belts in $90^{\circ}$ sitting position. We performed isometric measurements at $60^{\circ}$ of knee flexion. We requested the patients to contract the muscle that was evaluated for 15 -seconds twice in a 20 -second interval during the measurement. Following a 60-second resting period, we evaluated the patients with an isokinetic module. To increase compliance to the test, we conducted seven submaximal repetitions at $240 \%$ second, prior to the evaluation. Afterwards, we performed isokinetic test at $240^{\circ}, 180^{\circ}, 120^{\circ}$ and $60^{\circ}$ second angular velocities. For interpreting the results, we chose the data obtained from $60 \%$ second angular velocity. We repeated each set seven times at 60 -second intervals. Although we performed all evaluations on both knees, we used the data regarding the more symptomatic knee for statistical analysis.

The individuals underwent anteroposterior knee radiography with the knees in full extension. We graded each knee according to the KellgrenLawrence radiographic grading system that grades OA from 0 to $4 .{ }^{12}$ Ethical approval was obtained from the local ethics committee. All participants signed written informed consent before enrolment. The study was conducted in 
accordance with the principles of the Declaration of Helsinki.

\section{Statistical analysis}

We performed the statistical analysis using IBM SPSS statistical software version 22.0 (IBM Corporation, Armonk, NY, USA). We checked the normality of the variables. We used Mann Whitney- $\mathrm{U}$ and Kruskal-Wallis tests to analyze not-normally distributed continuous variables between the groups. We assessed the relationship between continuous variables by Spearman's rank correlation tests. We gave the results as mean \pm standard deviation. We considered $p$ values less than 0.05 as statistically significant. We classified the strength of correlations as no-very weak, weak-moderate, moderate-strong, and excellent when the correlation coefficient $(\mathrm{r})$ values were $0-0.24,0.25-0.49, \quad 0.50-0.74$, and 0.75-1.00, respectively. ${ }^{13}$

\section{RESULTS}

Mean body mass index (BMI) of the study population was $34.3 \pm 5.9 \mathrm{~kg} / \mathrm{m}^{2}$; none of the patients were within normal limits according to BMI. Additionally; 19.7\% ( $n=30), 59.2 \%(n=90)$, and $21.1 \%(\mathrm{n}=32)$ of the patients were morbid
Table 1. Demographic and clinical variables of study population

\begin{tabular}{|lc|}
\hline & Mean \pm SD \\
\hline Age (year) & $57.3 \pm 7.5$ \\
Weight $(\mathrm{kg})$ & $81.8 \pm 12.5$ \\
Height $(\mathrm{cm})$ & $154.4 \pm 7.4$ \\
Body mass index $\left(\mathrm{kg} / \mathrm{m}^{2}\right)$ & $34.4 \pm 5.9$ \\
Waist circumference $(\mathrm{cm})$ & $102.5 \pm 9.9$ \\
Symptom duration $(\mathrm{month})$ & $60.1 \pm 64.5$ \\
Duration of morning stiffness $(\mathrm{min})$ & $6.9 \pm 6.1$ \\
Comorbidity score & $7.0 \pm 3.5$ \\
Visual analog scale $(0-100 \mathrm{~mm})$ & $48.4 \pm 19.3$ \\
\hline SD: Standard deviation. & \\
\hline
\end{tabular}

obese $\left(B M I \geq 40 \mathrm{~kg} / \mathrm{m}^{2}\right)$, obese $\left(B M I \geq 30 \mathrm{~kg} / \mathrm{m}^{2}\right)$, and overweight $\left(\mathrm{BMI}=25-30 \mathrm{~kg} / \mathrm{m}^{2}\right)$, respectively. The demographic and clinical variables of the study population are given in Table 1 .

An analysis of the biomarkers according to BMI groups (morbid obese, obese, and overweight) revealed that only leptin level differed among the BMI groups $(p=0.000)$.

Biological markers and quadriceps strength measures (via MMT and isokinetic dynamometer) of the study population are given in Table 2 . Accordingly, the leptin level of females was

Table 2. Biological marker levels and quadriceps muscle strength measures of study population

\begin{tabular}{|c|c|c|c|}
\hline & Female $(n=130)$ & Male $(\mathrm{n}=22)$ & Total $(\mathrm{n}=152)$ \\
\hline \multicolumn{4}{|c|}{ Quadriceps muscle strength } \\
\hline MMT (lb) & $29.6 \pm 13.8^{*}$ & $43.7 \pm 15.5$ & $31.7 \pm 14.9$ \\
\hline \multicolumn{4}{|c|}{ Isometric (Peak torque, N-m) } \\
\hline Extensor $\left(60^{\circ}\right)$ & $78.5 \pm 22.1^{*}$ & $118.8 \pm 28.4$ & $84.4 \pm 27.0$ \\
\hline Flexor $\left(60^{\circ}\right)$ & $30.1 \pm 11.3^{*}$ & $49.2 \pm 18.3$ & $32.8 \pm 14.1$ \\
\hline \multicolumn{4}{|c|}{ Isokinetic (Peak torque, N-m) } \\
\hline Extensor $\left(60^{\circ}\right)$ & $64.0 \pm 20.1^{*}$ & $104.5 \pm 28.5$ & $69.8 \pm 25.7$ \\
\hline Flexor $\left(60^{\circ}\right)$ & $33.8 \pm 12.5^{*}$ & $53.5 \pm 19.7$ & $36.6 \pm 15.4$ \\
\hline \multicolumn{4}{|l|}{ Biological markers } \\
\hline Osteocalcin (ng/mL) & $18.1 \pm 8.6$ & $19.3 \pm 7.8$ & $18.3 \pm 8.5$ \\
\hline CTX-I (ng/mL) & $0.3 \pm 0.2$ & $0.3 \pm 0.2$ & $0.3 \pm 0.2$ \\
\hline CTX-II (ng/mL) & $1.1 \pm 0.8$ & $1.0 \pm 0.9$ & $1.1 \pm 0.8$ \\
\hline Leptin $(\mathrm{ng} / \mathrm{mL})$ & $23.8 \pm 13^{*}$ & $5.4 \pm 5.9$ & $21.1 \pm 13.8$ \\
\hline 25-(OH)D (ng/mL) & $16.1 \pm 11.2$ & $16.7 \pm 7.0$ & $16.2 \pm 10.7$ \\
\hline
\end{tabular}


Table 3. Correlation between biological markers and quadriceps muscle strength

\begin{tabular}{|c|c|c|c|c|c|}
\hline & $\begin{array}{l}\text { Osteocalcin } \\
\text { (ng/mL) }\end{array}$ & $\begin{array}{c}\text { CTX-I } \\
\text { (ng/mL) }\end{array}$ & $\begin{array}{c}\text { CTX-II } \\
\text { (ng/mL) }\end{array}$ & $\begin{array}{l}\text { Leptin } \\
\text { (ng/mL) }\end{array}$ & $\begin{array}{c}25(\mathrm{OH}) \mathrm{D} \\
(\mathrm{ng} / \mathrm{mL})\end{array}$ \\
\hline \multicolumn{6}{|c|}{ Quadriceps muscle strength } \\
\hline MMT (lb) & 0.16 & 0.14 & 0.18 & -0.22 & 0.10 \\
\hline \multicolumn{6}{|c|}{ Isometric (Peak torque, N-m) } \\
\hline Extensor $\left(60^{\circ}\right)$ & 0.01 & -0.05 & -0.09 & -0.20 & 0.09 \\
\hline Flexor $\left(60^{\circ}\right)$ & -0.07 & -0.11 & -0.02 & -0.18 & 0.10 \\
\hline \multicolumn{6}{|c|}{ Isokinetic (Peak torque, N-m) } \\
\hline Extensor $\left(60^{\circ}\right)$ & -0.02 & -0.08 & 0.00 & -0.18 & 0.03 \\
\hline Flexor $\left(60^{\circ}\right)$ & -0.08 & -0.06 & -0.12 & -0.16 & 0.05 \\
\hline
\end{tabular}

CTX-I: C-telopeptide of type I collagen; CTX-II: C-telopeptide of type II collagen; 25-(OH)D: 25-hydroxy vitamin D; MMT: Manual muscle test; N-m: Newton-meter; Values represent the Spearman's correlation coefficient $\left(\mathrm{r}_{\mathrm{s}}\right)$

significantly higher than males $(p=0.000)$. Besides, the quadriceps strength measure evaluated both by MMT and isokinetic dynamometer was significantly lower in females than that in males $(p=0.000)$.

The correlation between biological markers and quadriceps muscle strength was evaluated and no-very weak correlations were detected between biological markers and quadriceps muscle strength measures (Table 3).

We also carried out a correlation analysis to evaluate the relationship between quadriceps muscle strength and clinical data of the patients. The visual analog scale score of pain was weakly to moderately correlated with the parameters of isokinetic test ( $\mathrm{r}$ values ranging from -0.22 to -0.33 ). Comorbidity score showed a weak-moderate correlation with both MMT and isokinetic measures ( $\mathrm{r}$ values ranging from -0.27 to -0.32 ). When the correlation between quadriceps muscle strength and WOMAC scores was evaluated, weak-moderate correlations were found similarly ( $\mathrm{r}$ values ranging from -0.21 to -0.37$)$.

Table 4. Comparison of quadriceps muscle strength and biological marker levels according to Kellgren-Lawrence grades

\begin{tabular}{|c|c|c|c|c|c|}
\hline & Grade $1(n=76)$ & Grade $2(n=44)$ & Grade $3(n=21)$ & Grade $4(\mathrm{n}=11)$ & $p$ \\
\hline & Mean \pm SD & Mean \pm SD & Mean \pm SD & Mean $\pm S D$ & \\
\hline \multicolumn{6}{|c|}{ Quadriceps muscle strength } \\
\hline MMT (lb) & $32.4 \pm 15.3$ & $32.2 \pm 14.8$ & $30.9 \pm 15.2$ & $25.6 \pm 11.5$ & 0.533 \\
\hline \multicolumn{6}{|c|}{ Isometric (Peak torque, N-m) } \\
\hline Extensor $\left(60^{\circ}\right)$ & $88.3 \pm 27.5$ & $82.5 \pm 28.0$ & $81.5 \pm 21.8$ & $70.5 \pm 25.9$ & 0.137 \\
\hline Flexor $\left(60^{\circ}\right)$ & $34.5 \pm 15.2$ & $33.3 \pm 13.9$ & $29.1 \pm 10.5$ & $26.8 \pm 12.1$ & 0.288 \\
\hline \multicolumn{6}{|c|}{ Isokinetic (Peak torque, N-m) } \\
\hline Extensor $\left(60^{\circ}\right)$ & $72.1 \pm 28.4$ & $69.8 \pm 24.9$ & $67.0 \pm 18.8$ & $60.3 \pm 19.5$ & 0.536 \\
\hline Flexor $\left(60^{\circ}\right)$ & $38.3 \pm 16.6$ & $37.4 \pm 15.2$ & $34.0 \pm 11.8$ & $28.2 \pm 11.3$ & 0.226 \\
\hline \multicolumn{6}{|l|}{ Biological markers } \\
\hline Osteocalcin (ng/mL) & $17.9 \pm 8.8$ & $19.5 \pm 8.5$ & $16.9 \pm 5.9$ & $18.5 \pm 11.2$ & 0.672 \\
\hline CTX-I (ng/mL) & $0.3 \pm 0.2$ & $0.3 \pm 0.2$ & $0.3 \pm 0.1$ & $0.4 \pm 0.2$ & 0.515 \\
\hline CTX-II (ng/mL) & $1.0 \pm 0.9$ & $1.1 \pm 0.9$ & $1.1 \pm 0.9$ & $1.1 \pm 0.8$ & 0.935 \\
\hline Leptin $(\mathrm{ng} / \mathrm{mL})$ & $2.0 \pm 13.8$ & $21.2 \pm 13.2$ & $21.7 \pm 13.0$ & $28.2 \pm 17.7$ & 0.490 \\
\hline 25-(OH) D (ng/mL) & $16.7 \pm 11.5$ & $15.2 \pm 10.9$ & $16.6 \pm 9.1$ & $15.9 \pm 7.7$ & 0.541 \\
\hline
\end{tabular}


Finally, we interpreted the results according to the radiological grades of knee OA. Accordingly, quadriceps strength measures showed no significant difference among Kellgren-Lawrence grades. Regarding the biological markers, there was also no difference among Kellgren-Lawrence grades (Table 4).

\section{DISCUSSION}

In this study, we investigated quadriceps muscle weakness, which was thought to be the cause or the result of $\mathrm{OA},{ }^{1-3}$ in terms of its relationship with biological markers in patients with primary knee OA. We aimed to elucidate the relationship, if any, between quadriceps muscle strength and biological markers, as well as several clinical variables (pain scores and functional status) in patients with primary knee OA.

We detected no correlations between leptin, CTX-I, CTX-II, and osteocalcin levels and quadriceps muscle strength in the current study. The literature review produced one study investigating the relationship between biological markers and quadriceps muscle strength. ${ }^{14}$ This study by Røtterud et $\mathrm{a}^{14}$ focused on individuals with Kellgren-Lawrence grade $\geq 2$ knee OA and, conversely to our results, found that CTX-II was correlated with quadriceps muscle strength. Although leptin was suggested to be related with knee OA, ${ }^{15}$ our findings revealed a no-very weak negative correlation between leptin level and quadriceps muscle strength.

Mechanical and metabolic factors play a role in the development of $\mathrm{OA}$ and obesity is a well-known mechanical risk factor. In addition, there are also obesity-related metabolic factors including leptin and other adipokins. ${ }^{16,17}$ Leptin was shown to be related with increased prevalence and incidence of knee OA. Therefore, it was suggested that leptin could serve as a potential predictor of knee OA. ${ }^{15}$ Similarly, although not statistically significant, the present study showed that leptin levels were increasing as the radiological grade of knee OA increased. Concerning the cartilage biomarkers, urinary-CTX-II was not correlated with joint space narrowing in early knee radiographic OA. However, it was found to be correlated with cartilage defect severity measured by magnetic resonance imaging in the study by Ding et al. ${ }^{18}$ The authors concluded that urinary-CTX-II could be an early biomarker to show cartilage damage. ${ }^{18}$ In our study, we detected no significant difference among grades of knee OA regarding the other biological markers.

Although quadriceps muscle strength was not correlated with biological markers, it showed correlation with clinical variables including visual analog scale and WOMAC scores. Therefore, we may conclude that rather than biological markers, pain and functional status of patients might influence the strength of quadriceps muscle. There are a number of studies investigated the association of functional status with quadriceps muscle strength. Nevertheless, most of them appear to evaluate the impact of an intervention on quadriceps muscle strength. On the other hand, in consistence with our study, several studies so far have demonstrated the correlation between quadriceps strength and functionality. ${ }^{19,20}$ The results of these studies indicated that decreased isometric quadriceps muscle strength is related to knee symptoms and WOMAC functional disability. ${ }^{19,20}$

25-hydroxy vitamin D levels were not correlated with quadriceps muscle strength and patients' functional status in the current study. Similarly, Al-Jarallah et al. ${ }^{21}$ did not find any relationship between 25-(OH)D and the functional status of patients with primary knee OA in their crosssectional study. However, Barker et al. ${ }^{22}$ showed that decreased vitamin $\mathrm{D}$ levels are linked with diminished quadriceps functions. Contrary to the previous study, we could not find any correlation between $25-(\mathrm{OH}) \mathrm{D}$ level and quadriceps muscle strength in our study group. The most probable explanation for this difference is the limited number of patients with optimal vitamin D status, which avoids performing a comparative analysis.

The limitations of the current study are as follows: (i) the sample size was relatively small to suggest any correlation between biological markers and quadriceps muscle strength; (ii) the number of male patients was lower than that of females for making comparisons according to sex; and (iii) biological markers and quadriceps muscle strength could not be compared with healthy individuals as there was no control group. 
In conclusion, none of the studied biological markers were shown to have any deterministic effect on quadriceps muscle strength in patients with knee OA. On the other hand, despite the weak-moderate correlations of pain and functional status with quadriceps muscle strength, we may speculate that pain and functional disability might lead to quadriceps muscle weakness in patients with knee OA. Therefore, management of knee OA should primarily target pain control and functionality to prevent muscle weakness. Future longitudinal studies are needed to investigate the role of quadriceps muscle strength in knee $\mathrm{OA}$ and whether biomarkers can be used as a deterministic factor and contribute to the prognosis of knee OA.

\section{Declaration of conflicting interests}

The authors declared no conflicts of interest with respect to the authorship and/or publication of this article.

\section{Funding}

This study was funded by the Research Fund of the Cukurova University which had no involvement in the design, collection, analyses or reporting of the study (Trial Registration No. TF2013LTP23).

\section{REFERENCES}

1. Nelson AE, Jordan MJ. Osteoarthritis: epidemiology and classification. In: Hochberg MC, Silmon AJ, Smolen JS, Weinblatt EM, Weisman MH, editors. Rheumatology. 5th ed. Philadelphia: Elsevier; 2011. p. 1709-16.

2. Stitik TP, Kim JH, Stiskal D, Foye P, Nadler R, Wyss $\mathrm{J}$, et al. Osteoarthritis. In: WR Frontera, BM Gans, NE Walsh, LR Robinson, editors. Delisa's Physical Medicine and Rehabilitation. 5th ed. Philadelphia: Lippincott Williams \& Wilkins; 2010. p. 781-809.

3. Apold H, Meyer HE, Nordsletten L, Furnes O, Baste V, Flugsrud GB. Risk factors for knee replacement due to primary osteoarthritis, a population based, prospective cohort study of 315,495 individuals. BMC Musculoskelet Disord 2014;15:217.

4. Tuna S, Balc1 N. The relationship between radiological severity and functional status in patients with knee osteoarthritis. Clin Rheumatol 2014;33:667-70.

5. Segal NA, Zimmerman MB, Brubaker M, Torner JC. Obesity and knee osteoarthritis are not associated with impaired quadriceps specific strength in adults. PMR 2011;3:314-23.

6. Karsdal MA, Byrjalsen I, Bay-Jensen AC, Henriksen $\mathrm{K}$, Riis BJ, Christiansen C. Biochemical markers identify influences on bone and cartilage degradation in osteoarthritis--the effect of sex, Kellgren-Lawrence (KL) score, body mass index (BMI), oral salmon calcitonin (sCT) treatment and diurnal variation. BMC Musculoskelet Disord 2010;11:125.

7. $\mathrm{Ku} \mathrm{JH}$, Lee $\mathrm{CK}$, Joo BS, An BM, Choi SH, Wang $\mathrm{TH}$, et al. Correlation of synovial fluid leptin concentrations with the severity of osteoarthritis. Clin Rheumatol 2009;28:1431-5.

8. Kumm J, Tamm A, Lintrop M, Tamm A. The value of cartilage biomarkers in progressive knee osteoarthritis: cross-sectional and 6-year follow-up study in middleaged subjects. Rheumatol Int 2013;33:903-11.

9. Sowers MF, Karvonen-Gutierrez CA, Yosef M, Jannausch M, Jiang Y, Garnero P, et al. Longitudinal changes of serum COMP and urinary CTX-II predict X-ray defined knee osteoarthritis severity and stiffness in women. Osteoarthritis Cartilage 2009;17:1609-14.

10. Basaran S, Guzel R, Seydaoglu G, Guler-Uysal F. Validity, reliability, and comparison of the WOMAC osteoarthritis index and Lequesne algofunctional index in Turkish patients with hip or knee osteoarthritis. Clin Rheumatol 2010;29:749-56.

11. Sangha O, Stucki G, Liang MH, Fossel AH, Katz JN. The Self-Administered Comorbidity Questionnaire: a new method to assess comorbidity for clinical and health services research. Arthritis Rheum 2003;49:156-63.

12. Kellgren JH, Lawrence JS. Radiological assessment of osteo-arthrosis. Ann Rheum Dis 1957;16:494-502.

13. Özdemir O. Medikal istatistik. 1. Baskı. İstanbul: İstanbul Medikal Yayıncilik; 2006.

14. Røtterud JH, Reinholt FP, Beckstrøm KJ, Risberg MA, Arøen A. Relationship between CTX-II and patient characteristics, patient-reported outcome, muscle strength, and rehabilitation in patients with a focal cartilage lesion of the knee: a prospective exploratory cohort study of 48 patients. BMC Musculoskelet Disord 2014;15:99.

15. Karvonen-Gutierrez CA, Harlow SD, Mancuso P, Jacobson J, Mendes de Leon CF, Nan B. Association of leptin levels with radiographic knee osteoarthritis among a cohort of midlife women. Arthritis Care Res (Hoboken) 2013;65:936-44.

16. Anandacoomarasamy A, Fransen M, March L. Obesity and the musculoskeletal system. Curr Opin Rheumatol 2009;21:71-7.

17. Staikos C, Ververidis A, Drosos G, Manolopoulos VG, Verettas DA, Tavridou A. The association of adipokine levels in plasma and synovial fluid with the severity of knee osteoarthritis. Rheumatology (Oxford) 2013;52:1077-83.

18. Ding C, Garnero P, Cicuttini F, Scott F, Cooley $\mathrm{H}$, Jones G. Knee cartilage defects: association with early radiographic osteoarthritis, decreased cartilage volume, increased joint surface area and type II collagen breakdown. Osteoarthritis Cartilage 2005;13:198-205. 
19. Ruhdorfer A, Wirth W, Hitzl W, Nevitt M, Eckstein F. Association of thigh muscle strength with knee symptoms and radiographic disease stage of osteoarthritis: data from the Osteoarthritis Initiative. Arthritis Care Res (Hoboken) 2014;66:1344-53.

20. Ruhdorfer A, Wirth W, Eckstein F. Relationship between isometric thigh muscle strength and minimum clinically important differences in knee function in osteoarthritis: data from the osteoarthritis initiative. Arthritis Care Res (Hoboken) 2015;67:509-18.
21. Al-Jarallah KF, Shehab D, Al-Awadhi A, Nahar I, Haider MZ, Moussa MA. Are 25(OH)D levels related to the severity of knee osteoarthritis and function? Med Princ Pract 2012;21:74-8.

22. Barker T, Henriksen VT, Rogers VE, Aguirre D, Trawick RH, Lynn Rasmussen G, et al. Vitamin D deficiency associates with $\gamma$-tocopherol and quadriceps weakness but not inflammatory cytokines in subjects with knee osteoarthritis. Redox Biol 2014;2:466-74. 